\title{
Early Life Stress Inhibits Expression of a Novel Innate Immune Pathway in the Developing Hippocampus
}

\author{
Lan Wei', Arthur Simen', Shrikant Mane ${ }^{2}$ and Arie Kaffman*,' \\ 'Department of Psychiatry, Yale University School of Medicine, New Haven, CT, USA; ${ }^{2}$ Yale Center for Genomic Analysis, West Haven, CT, USA
}

\begin{abstract}
Childhood maltreatment represents a major risk factor for the development of numerous childhood psychopathologies that in many cases linger as chronic mental illnesses in adulthood. Exposing rodents or non-human primates to early life stress increases anxiety-like behaviors and impairs cognitive function in adulthood, suggesting that animal models may provide important insights into parallel developmental processes in humans. Using an unbiased genomic screen, we found that expression of lipopolysaccharide binding protein (LBP), a member of the innate immune system, is dramatically decreased in the hippocampus of pups exposed to early life stress. LBP levels peak in the normally developing hippocampus at a period of intense synaptic pruning, during which LBP is colocalized with the synaptic marker PSD95 and is found in close proximity to processes of microglia cells. Expression of LBP declines to low levels seen in adulthood at around postnatal day 30. Importantly, 30-day-old LBP knockout (k.o.) mice show increased spine density and abnormal spine morphology, suggesting that peak levels of LBP during the second and third weeks of life are necessary for normal synaptic pruning in the hippocampus. Finally, LBP K.o. mice show impaired hippocampal-dependent memory and increased anxiety-like behaviors in a manner that resembles that seen in animals exposed to early life stress. These findings describe a novel role for LBP in normal hippocampal development and raise the possibility that at least some of the behavioral sequelae of early life stress are mediated by reduced expression of LBP during a critical period of neurodevelopment.

Neuropsychopharmacology (2012) 37, 567-580; doi: I0. I038/npp.20I I.239; published online 12 October 201 I
\end{abstract}

Keywords: lipopolysaccharide binding protein; early life stress; mice; neurodevelopment; innate immune system; microarray

\section{INTRODUCTION}

Roughly 1.5 million children are abused or neglected each year in the United States and this alarming trend has been documented now for over 30 years (Kaffman, 2009). In the absence of effective interventions, many maltreated children can go on to develop a host of behavioral, emotional, cognitive, and medical sequelae that are chronic and often refractory to treatment (Gilbert et al, 2009; Kaffman and Meaney, 2007; Nemeroff et al, 2003; Zeanah et al, 2009). The relationship between early life stress and mental illness has been demonstrated using both retrospective and prospective studies (reviewed in Gilbert et al, 2009), and recent analysis of the National Comorbidity Replication Survey estimated that childhood maltreatment accounts for roughly a third of all adult psychopathology and almost half of all childhood psychopathologies (Green et al, 2010). The total cost associated with early life stress is estimated by

*Correspondence: Dr A Kaffman, Department of Psychiatry, Yale University School of Medicine, 300 George Street, Suite 901, New Haven, CT 065 I I, USA, Tel: + I 203785 6657, Fax: 203785 7357, E-mail: arie.kaffman@yale.edu

Received 31 May 2011; revised 4 September 2011; accepted 6 September 201 I the Institute of Medicine at 247 billion dollars annually, placing it at equal footing with the cost associated with all cancers combined. Despite the enormity of this problem, the neurodevelopmental details by which early adversity increases the vulnerability to mental illness are still poorly understood.

Similar outcomes have been reported in non-human primates and rodents exposed to early life stress, suggesting that animal models could provide important insights into this issue in humans (Kaffman, 2009; Kaffman and Meaney, 2007). In this regard, the availability of transgenic animals and genomic tools uniquely available in the mouse make it a particularly attractive model organism to study the molecular mechanisms by which early adversity modify neurodevelopment and adult behavior. However, the establishment of early life stress paradigms that are associated with robust and reproducible behavioral outcomes in the mouse has proven to be a challenging task (Millstein and Holmes, 2007; Priebe et al, 2005), explaining the paucity of studies that have used unbiased genomic tools and transgenic animals to study this issue. We spent a significant effort to develop a robust early life stress paradigm in the mouse and recently showed that brief daily separation (BDS), in the highly stress-reactive mouse 
strain $\mathrm{BALB} / \mathrm{cByj}$, is associated with prolonged elevation of corticosterone during the postnatal period, abnormal hippocampal development, and robust behavioral changes in adulthood that occur despite a compensatory increase in maternal care (Wei et al, 2010).

In studies reported here, we used a whole-genome microarray to identify genes whose expression in the developing hippocampus is altered by BDS. One of the most intriguing findings from the array was that exposure to BDS dramatically decreased expression of lipopolysaccharide (LPS) binding protein (LBP). We decided to further study the role of this gene in normal neurodevelopment because LBP was one of the most highly regulated genes we found on the array, yet practically nothing is known about its role in neurodevelopment or adult brain functioning. Moreover, LBP can deliver pathogens for phagocytosis via its ability to bind CD14 receptors found on macrophages (Zweigner et al, 2006), and phagocytosis of non-functional or redundant synapses, during a critical period, is necessary to establish an efficient wiring grid and normal adult brain functioning (Boulanger and Shatz, 2004; Dalmau et al, 1998; Stevens et al, 2007). Our findings suggest that LBP is a developmental protein whose expression rapidly decreases in the hippocampus in response to stress. Using LBP knockout (k.o.) mice, we show that LBP expression is necessary for normal hippocampal development and function in adulthood.

\section{MATERIALS AND METHODS}

\section{Animals}

Animals were housed in standard Plexiglas cages and kept on a standard $12: 12 \mathrm{~h}$ light-dark cycle (lights on at $0700 \mathrm{~h}$ ), constant temperature and humidity $\left(22^{\circ} \mathrm{C}\right.$ and $\left.50 \%\right)$ with food provided ad libitum. All studies were approved by the Institutional Animal Care and Use Committee at Yale University and were conducted in accordance with the recommendations of the NIH Guide for the Care and the Use of Laboratory Animals.

\section{Brief Daily Maternal Separation}

Sixty female and thirty male BALB/cByj mice, 6-8 weeks old, were purchased from Jackson Labs and allowed to acclimate for 2 weeks in our laboratory. Mating, BDS exposure, and observation of maternal behavior were done as described previously (Wei et al, 2010). In brief, animals were mated using a $2: 1$ female to male ratio with visibly pregnant dams placed individually in maternity cages with no nesting material. At birth (postnatal day $(\mathrm{PND})=0$ ), pups were culled to 6-8 pups per litter, and litters were randomly assigned to either BDS or control groups. The separation procedure occurred daily from PND 1 to 21 and was done from 1100 to $1140 \mathrm{~h}$. During the procedure, the dam was removed from the home cage and placed in a holding cage covered with fresh corncob bedding, and provided with food and water. Pups were transferred individually into a new cage covered with clean corncob bedding and placed at different corners of a $20.3 \times 27.9 \mathrm{~cm}$ standard cage. Cages were left undisturbed for $15 \mathrm{~min}$ at ambient temperature in the vivarium (ie, $22 \pm 2^{\circ} \mathrm{C}$ ) after which the pups were individually transferred to their home cage followed by the return of the dam. Maternal observations were conducted during four periods of 60 min each per day from PND 1 to 7. Cages in the control group were left undisturbed except for cage maintenance performed on PND 14. On PND 22, pups were weighed and housed in groups of 3-5 littermates of the same sex per cage.

\section{Acute Maternal Separation}

Acute maternal separation was done using three litters of PND 14 pups raised under control conditions (see above). Half of the pups ( $n=3-4$, both males and females) from each litter were kept undisturbed with the dam while the remaining pups were placed individually in a new cage with clean bedding maintained at ambient temperature in the vivarium (ie, $22 \pm 2^{\circ} \mathrm{C}$ ). After $3 \mathrm{~h}$ of maternal separation, all pups were rapidly killed to collect plasma and to harvest hippocampal tissue as described previously (Wei et al, 2010).

\section{LBP Offspring}

Homozygous LBP k.o. mice on a BALB/cJ background were purchased from Jackson and backcrossed to BALB/cByj mice for six generations. A group of 30 LBP heterozygous females and 15 LBP heterozygous males were mated using a $2: 1$ harem ratio. Visibly pregnant females were transferred to maternity cages and processed in the same way as described for the control group in the BDS procedure (see above). Pups were weaned, sexed, genotyped on PND 22, and housed in groups of 3-5 same sex littermates per cage until used for behavioral testing or analysis of dendritic morphology.

\section{Behavioral Studies}

All behavioral work was done between 1300 and $1700 \mathrm{~h}$ under dim lighting conditions (30 Lux in the arena) because of the high anxiety and sensitivity to bright light in this strain. Only adult males (PND 90-110) were included in the study because our preliminary studies and work from other laboratories have shown that behavioral sequelae of early life stress in mice, especially those related to anxiety-like behavior, are more robust in male offspring (Berrebi et al, 1988; Denenberg et al, 1981; Kikusui et al, 2006; Millstein et al, 2006; Parfitt et al, 2004; Ren-Patterson et al, 2006; Wei et al, 2010). No $>1-2$ offspring from the same litter were used and at least eight litters were represented in each group tested. To assess the behavioral effects of BDS, male mice ( $n=10$ for each condition) were assessed in this order: open field, step down, and Morris water maze. A second cohort ( $n=8$ for each condition) was tested in the dark-light test followed by the object recognition test. Behavioral testing in LBP k.o. offspring was done in the following order: open field, step down, dark-light, and object recognition.

\section{Morris Water Maze}

A $10 \times 10 \mathrm{~cm}$ platform was made invisible by immersing it in a pool (1 $\mathrm{m}$ in diameter) filled to $1 \mathrm{~cm}$ above platform 
level with water $\left(22 \pm 1^{\circ} \mathrm{C}\right)$ that was made opaque by the addition of a water-based white powder (white powder tempera \#427217). The pool was placed in the middle of a small room $(3.66 \times 3.66 \mathrm{~m})$ with large visual cues present on its walls. The mouse was introduced into the pool at a randomly assigned starting point (ie, East and West) and allowed to swim until it found the platform or the trial terminated after $2 \mathrm{~min}$. There were four trials per day that were spaced roughly $30 \mathrm{~min}$ apart, with the daily latency to escape calculated as the average across the four trails. Animals were initially tested in the cued-platform condition in which the position of the platform changed daily but was made visible using an attached flag. In the second phase of testing (ie, hidden platform), the position of the platform was kept constant across the 8 days of testing, but the visual cue that marked the position of the platform was removed requiring the animal to use external cues to locate the hidden platform.

\section{Object Recognition}

Adult male mice were habituated to an opaque chamber $(45.7 \times 24 \mathrm{~cm})$, where two identical objects were placed at opposite sides of the cage for 15 min on 2 consecutive days. On the third day, the mouse was returned to the same chamber except that one of the objects was replaced with a novel object and the session was videotaped for $5 \mathrm{~min}$. The position and the identity of the novel object were randomized to eliminate object and position biases. The amount of time the mouse explored each object was scored by a rater who was blind to the mouse's genotype and developmental history, and was used to calculate \% preference index (\% PI). The \% PI was calculated as follow: $\% \mathrm{PI}=($ time exploring novel object $\times 100) /$ time exploring both objects.

\section{Open-Field Exploration}

This was done as described previously (Wei et al, 2010). In brief, individual adult male mice were allowed to freely explore a black $50 \times 50 \mathrm{~cm}$ Plexiglas arena for $5 \mathrm{~min}$. The distance traveled and the amount of time the mouse spent in the inner $15 \mathrm{~cm}$ area were determined using the EthoVision tracking system (Noldus Information Technology).

\section{Step-Down Test}

This was done as described previously (Wei et al, 2010). In brief, adult male mice were placed on a small 96-well plate covered with a rubber sheath to provide good grip for the animals. The latency to step off the platform (all four legs) was recorded by an observer who was blind to the developmental history of the animal.

\section{Dark-Light Choice}

A $44 \times 20 \times 20 \mathrm{~cm}$ Plexiglas box was partitioned so that two thirds of the length were white walled and illuminated with a light source at 15 Lux, while one third had black walls and was completely darkened (1 Lux). A small opening connected the two compartments. Mice were initially placed in the white compartment and allowed to explore the box freely for $10 \mathrm{~min}$ while being videotaped. An observer who was unaware of the genotype of the animal scored the total number of transitions between the two compartments, and time spent in the light compartment.

\section{RNA Harvesting and Microarray Work}

In all, 6 BDS and 6 control male pups, from 12 independent litters, were killed $3 \mathrm{~h}$ after the last maternal separation on PND 21. Hippocampal RNA was harvested and processed as described before (Wei et al, 2010). RNA samples were then processed by the Yale Keck Neuroscience Array Center and hybridized to the Illumina whole-genome mouse platform (V 1.1). For more details on sample processing, data analysis, and array results see Supplementary Information.

\section{Quantitative PCR}

Sample processing and analysis were done as described previously (Wei et al, 2010). LBP levels in the hippocampus were assessed using LBP-2F: CGTGGGCAGTACGAGTTTCA TAGC and LBP-2R: AGGAGCCATGAAGTTTCAGGAAGG primers that target the $5^{\prime}$ end of LBP instead of the $3^{\prime}$ of the gene that was detected by the array probe. TBP-F-1: AAAG GGAGAATCATGGACCAGAACAA and TBP-R-1: TGGACT AAAGATGGGAATTCCAGGAG primers were used to assess levels of the TATA binding protein (TBP) used as an internal control. Efficiency of all primers was confirmed to be $100 \pm 10 \%$ and the $2^{\Delta \Delta \mathrm{CT}}$ method was used (Pfaffl, $2001)$ to calculate relative mRNA levels. The calibrator groups used in different experiments are specified in figure legends.

\section{In Situ Hybridization}

In situ hybridization was performed on $14-\mu \mathrm{m}$ sagittal cryosections as described previously (Duric et al, 2010). LBP antisense probe was made using the following PCR primers: LBP-ISH-3F: $5^{\prime}$-aaggccttccgtcccttcac- $3^{\prime}$ and LBPISH-3R: 5 -CCAAGCCTTCTAATACGACTCACTATAGGGAGA cagcatcccggtaaccttgc- $3^{\prime}$ (sequence for T7 promoter shown in capital letters). This probe hybridizes to LBP mRNA and was used to quantify expression levels at different subfields of the hippocampus. LBP sense probe (ie, negative control) was made using LBP-ISH-5F: 5'-CCAAGCCTTCTAATACG ACTCACTATAGGGAGAaaggccttccgtcccttcac- $3^{\prime}$ and LBPISH-5R: $5^{\prime}$-cagcatcceggtaaccttgc- $3^{\prime}$. Both pairs of primers produced a single product with the expected 250-bp size that was sequenced to confirm its specificity. PCR products were then used as templates to generate ${ }^{35} \mathrm{~S}$-labeled radio cRNA probes that were incubated with the slides. Slides were then washed extensively, dried, and exposed to Kodak MR autoradiographic film. Images were then digitized and analyzed using Image J software (ImageJ 1.42, NIH).

\section{Plasma Corticosterone Levels}

Plasma corticosterone levels were assessed using an ${ }^{125}$ I-RIA kit (MP Biomedicals, Cat. No. 07-120102) as described previously (Wei et al, 2010). 


\section{Immunohistochemistry and Microscopy}

PND 21 male pups were anesthetized with Pentobarbital $(85 \mathrm{mg} / \mathrm{kg})$ and transcardially perfused with cold PBS/ heparin (50 units $/ \mathrm{ml}$ ) solution followed by $4 \%$ paraformaldehyde. Brains were then postfixed with $4 \%$ paraformaldehyde overnight and equilibrated in a $30 \%$ sucrose solution. Forty-micron coronal sections were collected using a freezing sliding microtome and incubated overnight at $4{ }^{\circ} \mathrm{C}$ with goat anti-LBP antibodies $(1: 100$, Santa Cruz, Cat. \#sc-14668) and either rabbit anti-PSD95 (1:200, Zymed Laboratories, Cat. \#51-6900) or rabbit anti-Iba1 (1:250, Wako, Cat. \#019-19741). Similar patterns of LBP staining were seen with mouse anti-LBP $(1: 100$, Enzo Life Sciences, Cat. \#ALX-804-502), goat anti-LBP ( $1: 100$, Santa Cruz, Cat. \#sc-14666), and rabbit anti-LBP antibodies $(1: 250$, Cell Sciences, Cat. \#CPL105). Slices were then washed and incubated with the appropriate fluorescently labeled DyLight antibodies ( $1: 250$, Jackson ImmunoResearch) and mounted in Vectashield mounting medium (Vector Laboratories). High-resolution ( $1024 \times 1024$ pixels) confocal images were taken using a Zeiss LSM 510 Meta microscope under a $63 \times$ apochromat water immersion lens (1.2 NA).

\section{Western Blot}

A total of $50 \mu \mathrm{g}$ of hippocampal lysate was separated on a $10 \%$ SDS-PAGE gel, transferred onto a $0.45-\mu \mathrm{m}$ nitrocellulose membrane, and incubated overnight at $4{ }^{\circ} \mathrm{C}$ with goat anti-LBP ( $1: 1000$, Santa Cruz, Cat. \#sc-14668) and rabbit anti- $\beta$ tubulin antibodies $(1: 20000$, Abcam Cat. \#ab6046) in Li-Cor blocking solution containing $0.1 \%$ Tween-20. The membrane was washed extensively with PBS and incubated with fluorescently labeled secondary antibodies (Li-Cor). LBP levels were quantified using an Odyssey infrared imaging system (Li-Cor Biosciences) with $\beta$-tubulin levels used to normalize loading.

\section{Golgi Staining and Morphological Analysis}

PND 30 LBP k.o. and wild-type littermate males $(n=5$ for each genotype) were rapidly decapitated. Brains were harvested and cut at midline. The left hemisphere was stained with rapid GolgiStain kit (FD Neurotechnologies), cut at $100-\mu \mathrm{m}$ sagittal sections on a cryostat, and processed according to the manufacturer's instructions. Stained slides were coded to ensure that morphological analysis was conducted by an observer who is blind to the animals' genotype. Morphological analysis followed a previously described protocol (Champagne et al, 2008) with the following modifications: CA1 pyramidal cells, located in the dorsal hippocampus ( $n=5$ for each animal), that were fully impregnated and free of neighboring cells or cellular debris were randomly selected for analysis. Cells were traced using the shaft method in Neurolucida 8.0 (MBF) to obtain the dendritic length and the number of branch points. Spine density was determined by counting the number of spines on 3-secondary and 3-tertiary dendritic segments that were each $30-40 \mu \mathrm{m}$ in length. Spine density from each individual branch was calculated by dividing the total number of spines counted by the branch length and averaged across the three measurements to obtain the spine density of the secondary and tertiary apical branches for each animal.

\section{Statistical Analysis}

The data were carefully screened for inaccuracies, outliers, normality and homogeneity of variance. Repeated measures analysis of variance (ANOVA) was used to analyze the effect of BDS on swim speed, \% time in the periphery, and latency to find the platform in the Morris water maze. Unpaired Student's $t$-tests were used in all cases where two independent groups were compared, and paired Student's $t$-tests were used to compare the dendritic morphology between LBP wild-type and k.o. littermates. A two-way ANOVA was used to assess the effects of BDS and age on LBP levels in the hippocampus. Significant main effects and interactions were followed by simple-effect analysis for each age. One-way ANOVA was used to assess the effect of LBP genotype on anxiety-like behaviors with Tukey-HSD post hoc analysis used to follow significant main effect of genotype. The PASW Statistics 18 software (IBM) was used for statistical analysis with $p<0.05$ considered as significant.

\section{RESULTS}

\section{Exposure to BDS Is Associated with Robust and Reproducible Behavioral Changes in Adulthood}

Using an independent cohort of animals, we replicated our previous finding showing that exposure to BDS increases maternal care during the first week of life and is associated with prolonged elevation of corticosterone levels in 14-dayold pups (Supplementary Figure S1). Exposure to BDS blunts the hippocampal growth spurt seen in normal 14-day-old pups (Wei et al, 2010) raising the possibility that this developmental insult may impair normal hippocampal function in adulthood. We tested this issue by assessing performance of adult male offspring exposed to $\mathrm{BDS}$ or control condition in the Morris water maze and the object recognition tests.

Repeated measures ANOVA showed a significant effect of experimental condition (ie, BDS $v s$ control, $F(1,18)=16.9$, $p=0.001)$ and a significant interaction between experimental condition and days of training $(F(7,126)=4.83$, $p=0.002$ ) on latency to find a hidden platform, demonstrating that exposure to BDS impairs performance in the Morris water maze in adulthood (Figure 1a). There were no differences in swimming speed $(F(1,18)=1.68, p=0.21)$ or percent time spent near the edges of the swimming pool $(F(1,18)=1.25, p=0.28)$ between BDS and control mice, indicating that differences in their ability to locate the hidden platform were not due to altered swimming capability or thigmotaxis. Similarly, there were no differences between the groups in their abilities to locate a cued platform (Figure $1 \mathrm{~b} ; F(1,18)=0.761, p=0.394$ ), indicating that BDS does not effect visual acuity or the ability to escape the water. Adult male mice exposed to BDS also showed impairment in their ability to preferentially explore a novel object in the object recognition task (Figure 1c; $t(14)=3.91$, $p=0.002)$. Both BDS and control mice spent similar amounts of time exploring objects during the training 

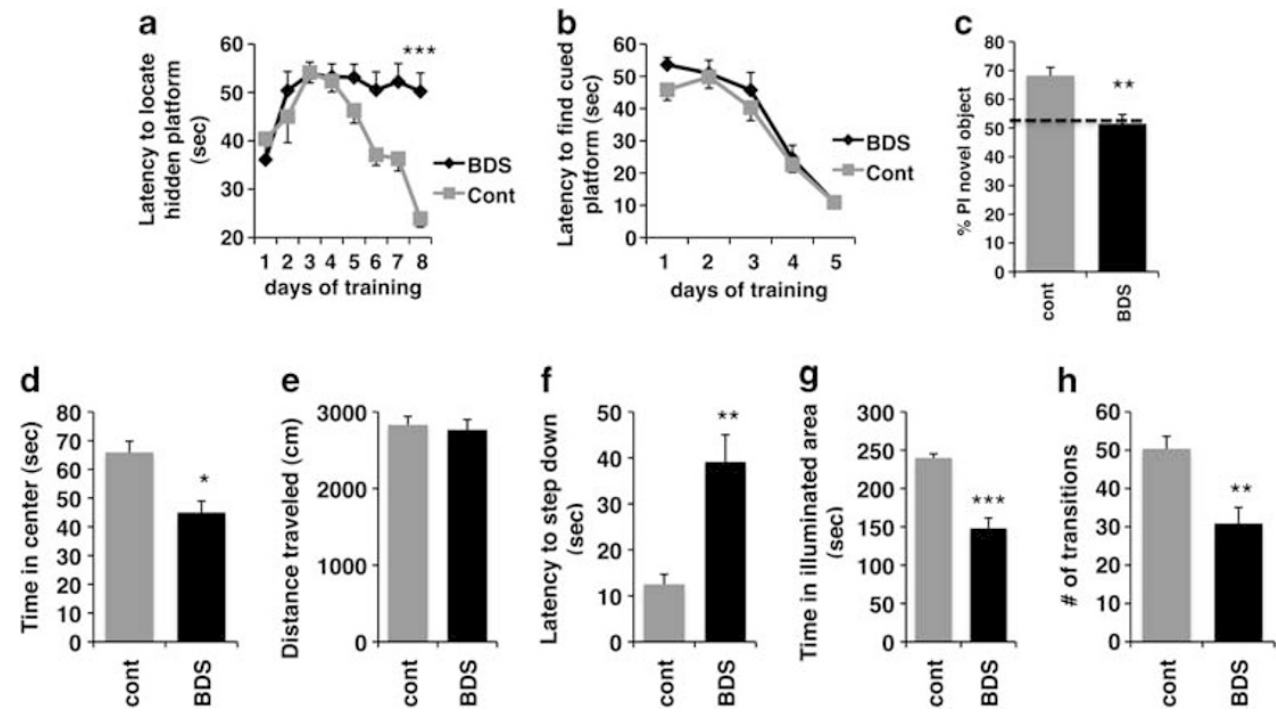

Figure I Exposure to BDS is associated with impaired hippocampal function and increased anxiety-like behavior in adulthood. Adult male offspring exposed to BDS showed impairment in their ability to locate a hidden platform (a) but not a visibly cued platform (b) in the Morris water maze. Exposure to BDS impaired preference to explore the novel object in the object recognition test (c). \% Preference index (PI) was calculated as a percentage of time the mouse explored the novel object divided by the total time exploring both objects. Offspring exposed to BDS spent less time in the center of the open field (d), but traveled the same distance as offspring exposed to the control condition (e) in the open-field test. Exposure to BDS increased latency to step down in the step-down test ( $f$ ), and decreased time in the illuminated area (g) and number of transitions (h) in the dark-light test; $n=8-10, P N D$ 90-I I0 adult males in each group. Results are expressed as mean values \pm SEM, repeated measure ANOVA for $(a, b)$ and unpaired Student's $t$-test for (c-h), * $p<0.05$, *** $p<0.0$ l, **** $p<0.001$.

session $(t(14)=0.19, p=0.85)$, suggesting that the failure to preferentially explore the novel object was not due to inappropriate object investigation during the training session. These findings are consistent with previous work showing that exposure to stress early in life is associated with impaired hippocampal-dependent learning in adulthood (Aisa et al, 2007; Fabricius et al, 2008; Rice et al, 2008).

Consistent with our previous work, exposure to BDS increased anxiety-like behavior in adulthood (Wei et al, 2010). BDS offspring spent less time exploring the center of the open field compared with control mice (Figure 1d; $t(1,18)=2.69, p=0.015)$, but there was no difference between the groups in the total distance traveled (Figure 1e), indicating that the difference in exploring the center was not due to changes in locomotor activity. In addition, adult offspring exposed to BDS early in life showed increased latency in the step-down test (Figure 1f; $t(1,18)=2.85, p=0.001)$, spent less time in the illuminated area (Figure $1 \mathrm{~g} ; t(14)=6.36, p<0.0005$ ), and made fewer transitions (Figure $1 \mathrm{~h} ; t(14)=3.68, p=0.002$ ) in the darklight test. These studies extend previous work showing that exposure to BDS, in this highly stress-reactive strain, is associated with robust and reproducible behavioral changes in adulthood.

\section{LBP Expression in the Developing Hippocampus Is Downregulated by BDS}

To further characterize the effect of BDS on hippocampal development, we harvested RNA from the hippocampus of 21-day-old male pups (BDS, $n=6$; control, $n=6$, from 12 different litters) and used a whole-genome microarray screen to identify genes whose expression is modified by BDS (see Supplementary Table S1 in Supplementary Information for a complete list of genes identified). One of the most intriguing findings from the array was that levels of LBP were dramatically reduced in BDS compared with control pups (Figure 2a; $p<0.0005$, $Q=0.099$ ). To confirm the array findings, we assessed levels of LBP using a qPCR amplification method that targeted the $5^{\prime}$ end of LBP instead of the $3^{\prime}$ end sampled by the array. Levels of LBP assessed by $\mathrm{qPCR}$ were highly correlated with levels found on the array $(r=0.97$, $p<0.0005)$ and confirmed that LBP levels were significantly lower in 21-day-old pups exposed to BDS (Figure 2b). In situ hybridization showed that expression of LBP in 21day-old male pups was mainly confined to the hippocampus and the choroid plexus (Supplementary Figure S2 in Supplementary Information) and that exposure to BDS decreased expression of LBP in the dentate gyrus and CA3 (Figure $2 \mathrm{c}$ and $\mathrm{d}$ ). Together, these data demonstrate that levels of LBP mRNA are downregulated in 21-day-old pups exposed to BDS.

Next, we tested whether exposure to BDS modifies LBP expression earlier in development and whether differences in its expression persist into adulthood. To address these issues, levels of LBP were determined by qPCR in BDS and control male mice on PND 8, 14, 21, and 120 (a total of 55 animals, with $n=6-10$ offspring in each time point). Two-way ANOVA showed a main effect of BDS exposure $(F(1,47)=6.30, \quad p=0.016), \quad$ a main effect of age $(F(1,47)=12.05, p<0.0005)$, and an interaction between BDS and age $(F(1,47)=4.59, p=0.007$; Figure $3 a)$. The pattern of LBP expression in the control group suggested that LBP is a developmental gene whose expression in the 

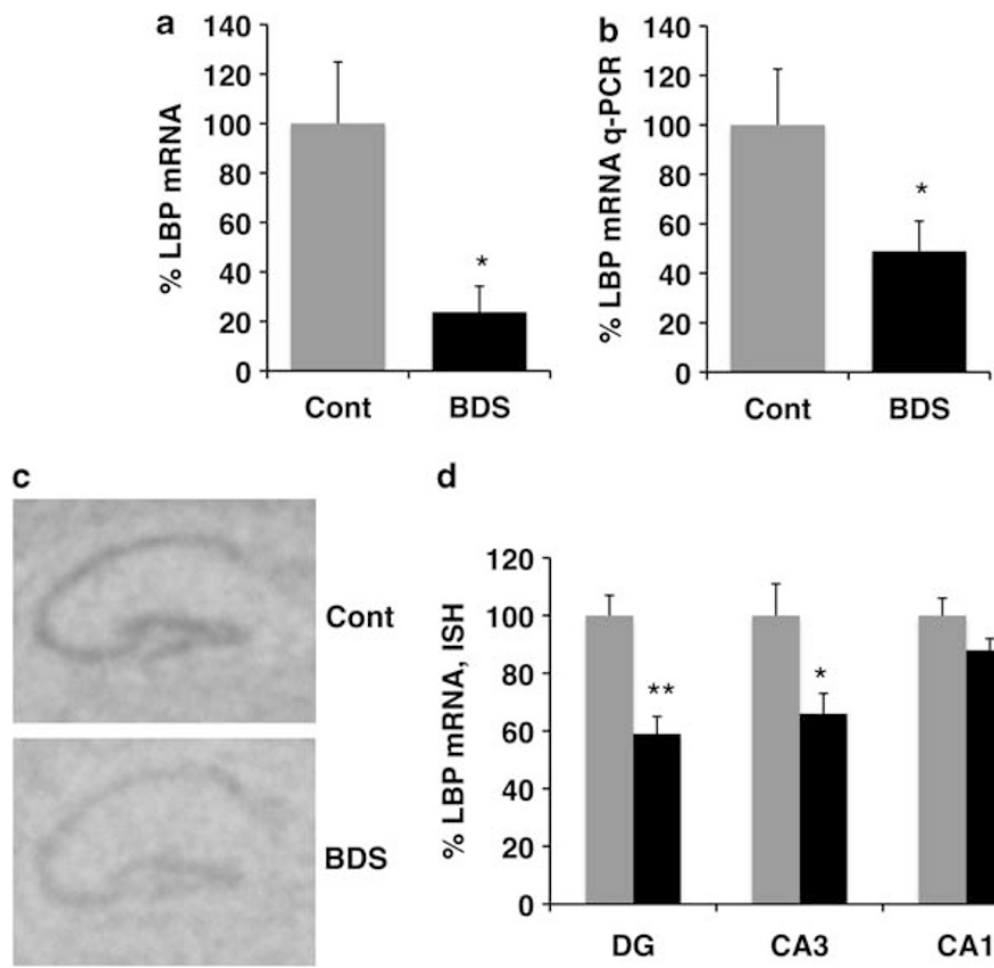

d

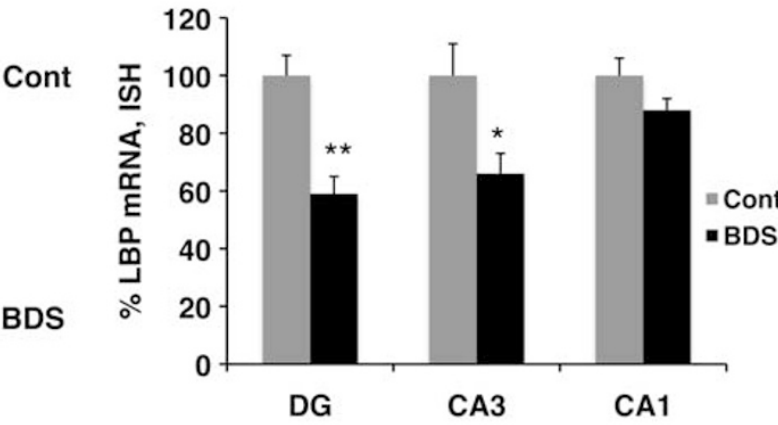

Figure 2 BDS decreases expression of LBP in 2 I-day-old pups. (a) LBP mRNA levels obtained from the mouse illumina whole-genome array (BDS $n=6$, control $n=5$ male pups). (b) Quantitative PCR confirming the array results. Representative autoradiographs (c) and quantitative analysis (d) of LBP mRNA levels detected by in situ hybridization in 21 -day-old pups exposed to BDS or control condition ( $n=5$ for each condition). Y axis shows LBP mRNA levels as a percentage of the control group. Bars and error bars represent mean values \pm SEM, unpaired Student's $t$-test for (a, b) repeated measure ANOVA for ( $d$ ), $* p<0.05, * * * 0.01$

hippocampus peaks during the second and third weeks of life and then declines in adulthood. To better characterize this developmental pattern, we compared levels of LBP in PND 5, 14, 21, 30, and 45 male offspring raised under the control condition ( $n=4-5$ for each time point). This analysis confirmed that levels of LBP rise during the second and third weeks of life followed by a decline to adult levels at around PND 30 (Figure 3b).

The findings that BDS decreased levels of LBP at PND 14 and 21, but not at PND 8 when the hypothalamic pituitary adrenal (HPA) axis has not yet matured (Levine, 1994; Wei et al, 2010), or in adulthood when stress is no longer present, suggested that exposure to stress may be necessary to inhibit LBP expression in the hippocampus. We, therefore, tested whether a single, 3-h maternal separation exposure was sufficient to decrease levels of LBP in 14day-old pups. Acute maternal separation increased plasma corticosterone levels in the pups (Figure 4a; $t(14)=3.72$, $p=0.002$ ), decreased LBP mRNA (Figure $4 \mathrm{~b} ; t(14)=2.20$, $p=0.045$ ) and protein levels in the hippocampus (Figure 4c and $\mathrm{d} ; t(6)=3.764, p=0.009$ ). Importantly, levels of LBP mRNA in the hippocampus were highly correlated with corticosterone plasma levels $(r=-0.89, p=0.04)$, supporting the notion that increased corticosterone during early development leads to a rapid decrease in LBP mRNA levels in the hippocampus. Acute stress had no effect on LBP levels in the plasma (Figure 4e; $t(6)=0.58, p=0.58$ ), suggesting that acute stress regulates LBP differently in the hippocampus and the plasma.

\section{LBP Is Required for Normal Synaptic Development in} the Hippocampus

Immunohistochemical staining of sections from PND 21 pups with purified antibodies against LBP revealed a punctate pattern of staining, throughout the hippocampus, reminiscent of synaptic proteins (Stevens et al, 2007). This pattern was seen using three different antibodies against LBP and was not present in the LBP k.o. mice (Supplementary Figure S3; Supplementary Information). Using confocal microscopy, we found that the LBP puncta were in close proximity to immunostaining of the synaptic marker PSD95, suggesting that LBP is localized to the developing synapse (Figure 5a). Double labeling with anti-LBP and anti-Iba-1 antibodies (ie, a marker of microglia cells) showed that many LBP puncta were in close proximity to processes of microglia cells (Figure $5 \mathrm{~b}$ ), consistent with previous work showing that LBP is able to interact with CD14 surface molecules present on microglia cells (Peterson et al, 1995).

Next, we compared dendritic morphology in PND 30 LBP k.o. and wild-type littermates, an age in which LBP levels decline to their adult levels (Figure $3 \mathrm{~b}$ ). Spine densities of both secondary (Figure $6 a ; t(4)=4.35 p=0.012$ ) and tertiary (Figure $6 \mathrm{~b} ; t(4)=5.13 p=0.007$ ) apical dendrites were increased in PND 30 LBP k.o. compared with their wild-type littermates ( $n=5$ for each genotype). Importantly, many of the dendritic spines in LBP k.o. mice were abnormally shaped (Figure 6c), consistent with a defect in 
a
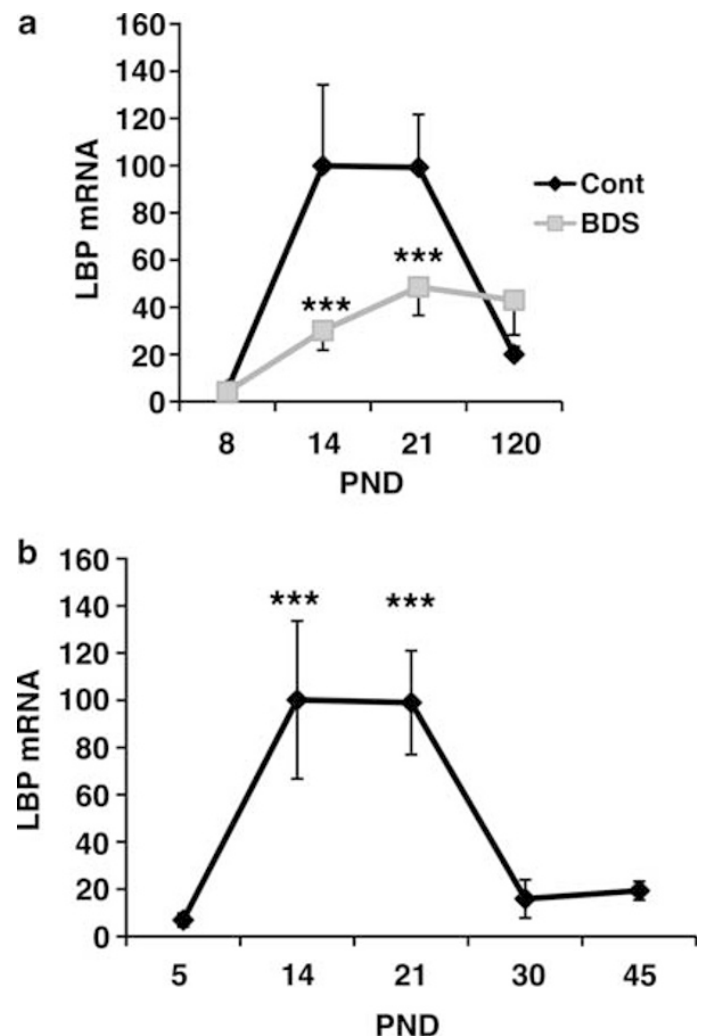

Figure $3 \mathrm{LBP}$ is a developmental gene whose expression is inhibited by BDS. (a) Quantitative PCR showing the effect of BDS on LBP mRNA levels in the hippocampus of PND 8, 14, 21, and 120 mice (a total of 55 male offspring, with $n=6-10$ mice in each time point). (b) Quantitative PCR assessing LBP mRNA levels in the hippocampus using a second cohort of control male mice assessed on PND 5, 14, 21, 30, and $45(n=4-5$ for each time point). PND 21 control group was used as the calibrator group (Pfaffl, $200 \mathrm{I}$ ) in both panels. Results are expressed as mean values \pm SEM, twoway ANOVA (a) and one-way ANOVA (b) $* * * * p<0.001$.

removing non-functional synapses in these mice. There were no differences between k.o. and wild-type littermates in the overall dendritic length (Figure 6d; $t(4)=0.74$ $p=0.5$ ) or number of branches (Figure $6 \mathrm{e} ; t(4)=0.77$ $p=0.48)$. LBP k.o. offspring were indistinguishable from their wild-type littermates in terms of appearance or weight during development (ie, PND 14, 21, and 30,p>0.05), indicating that the absence of this gene does not cause any gross developmental abnormalities. These data indicate that expression of LBP is necessary for normal synaptic development in the hippocampus.

\section{LBP k.o. Mice Show Behavioral Abnormalities That Resemble Those Seen in Animals Exposed to Early Life Stress}

The abnormal dendritic morphology seen in juvenile LBP k.o. mice raised the possibility that LBP expression might also be necessary for hippocampal-dependent function in adulthood. We assessed this issue by comparing performance in the object recognition task in adult LBP homozygous k.o., LBP heterozygous, and wild-type male littermates (ie, PND 90-110, $n=9-11$ males per genotype). Wild-type offspring showed the normal tendency to preferentially explore a novel object compared with a familiar object (Figure 7a; one-sample Student's $t$-test, $t(10)=6.53 p<0.0005$ ). In contrast, homozygous k.o. and heterozygous littermates showed no preference to explore the novel object, consistent with impaired hippocampaldependent memory in these animals (Figure $7 a, p>0.05$ ). All three groups spent similar amounts of time exploring the two objects $(F(2,27)=1.05, p=0.36)$, indicating that these differences were not due to a non-specific decreases in exploratory behavior. We also found that LBP deletion resulted in a dose-dependent decrease in the time spent in the center of the open field (Figure $7 \mathrm{~b} ; F(2,55)=3.28$, $p=0.045$ ), but had no effect on the total distance traveled in the open field (Figure $7 c ; p>0.05$ ). These results demonstrate that LBP deletion does not impair locomotor activity, and that the decreased tendency of LBP k.o. mice to explore the center of the open field is not due to a general reduction in locomotor activity. Finally, LBP mutant mice showed increased anxiety-like behavior in the step-down test (Figure $7 \mathrm{~d} ; F(2,55)=20.03, p<0.0005)$ and the dark-light test (Figure 7e; $F(2,55)=4.69, p=0.013$ and Figure 7f; $F(2,55)=5.25, p=0.008)$. Together, these results demonstrate that LBP k.o. mice show behavioral abnormalities that resemble those seen in mice exposed to BDS (compare Figures 1 and 7).

\section{DISCUSSION}

We previously showed that exposure to BDS in the highly stress-reactive $\mathrm{BALB} / \mathrm{cByj}$ strain is associated with robust and reproducible increase in anxiety-like behaviors in adulthood despite the ability of this manipulation to increase postnatal maternal care (Wei et al, 2010). To the best of our knowledge, this is the first example demonstrating increased anxiety-like behavior in animals receiving higher levels of postnatal maternal care. We propose that this unexpected yet robust finding is due to the fact that BDS is associated with an unusually prolonged elevation of corticosterone levels during the second and third weeks of life. We suspect that this outcome is due to a combination of factors that includes the high-stress reactivity that characterizes this strain (Shanks et al, 1990; Zaharia et al, 1996) and specific technical details associated with our BDS procedure (eg, the absence of nesting material, placing the pups apart at ambient temperature during the separation period, and the presence of a researcher that monitors maternal care). This mild form of early life stress is then responsible for the neurodevelopmental effects of BDS (eg, decreased DNA and RNA content of the developing hippocampus) in a manner that overrides the increased postnatal maternal care associated with this procedure (for a more detailed discussion of this issue, see Wei et al, 2010).

In studies reported here, we replicated our earlier findings and extended them to show that exposure to BDS is also associated with a defect in hippocampal-dependent learning. These findings are consistent with previous work showing that exposure to early life stress is associated with poor hippocampal function (Aisa et al, 2007; Fabricius et al, 2008; Kalinichev et al, 2002; Murgatroyd et al, 2009; Rice et al, 2008) and increased anxiety-like behavior in rodents (George et al, 2011; Muhammad and Kolb, 2011; Romeo et al, 2003). 

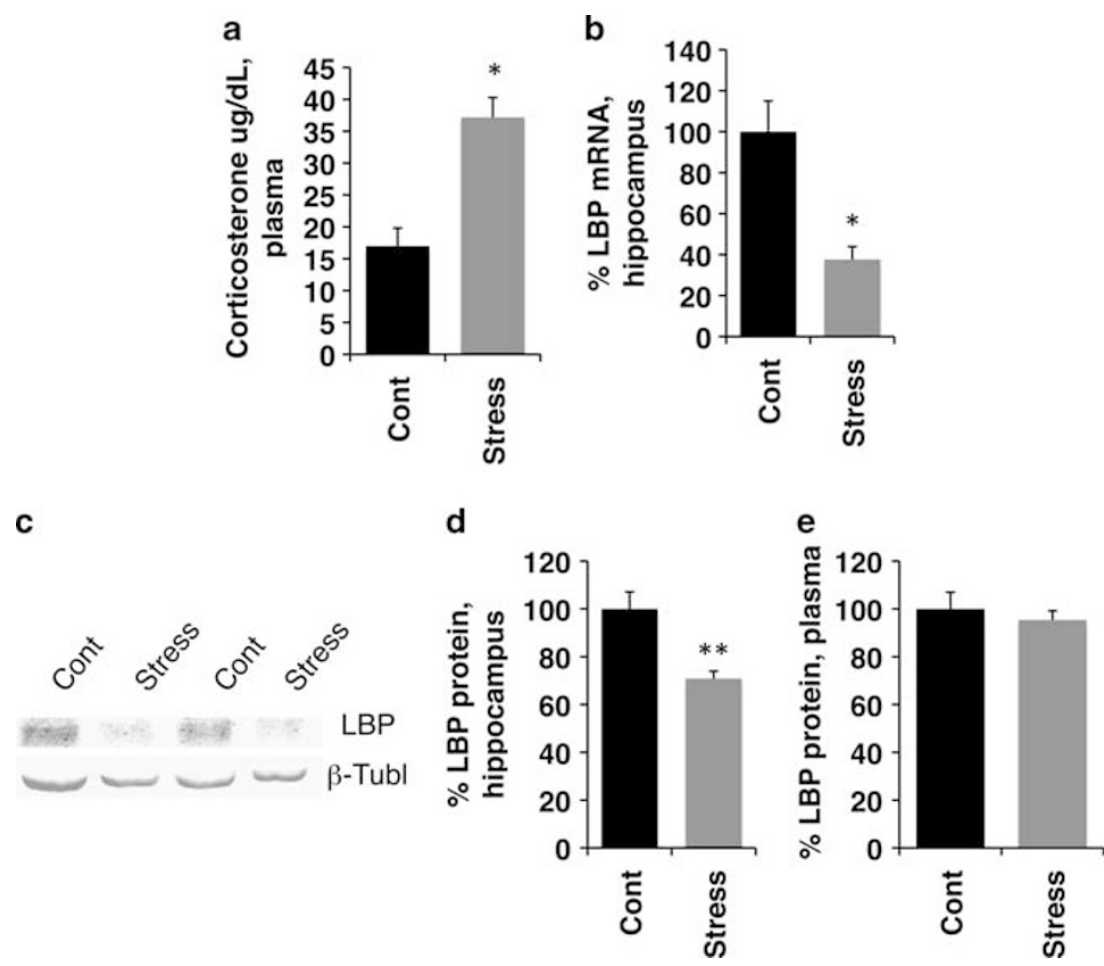

Figure 4 Acute stress is sufficient to decrease LBP in the hippocampus but not in the plasma. Exposure to a single 3-h maternal separation period in I4day-old pups caused a significant increase in plasma corticosterone (a) and decreased LBP mRNA in the hippocampus (b). Representative western blot (c) and quantitative analysis (d) showing that LBP levels are decreased in the hippocampus of animals exposed to $3 \mathrm{~h}$ of maternal separation. This manipulation did not affect levels of LBP in the plasma (e). For antibody specificity, see Supplementary Figure S3. N=5 in each condition (using three different litters). Results are expressed as mean values \pm SEM, $* p<0.05$, ** $p<0.01$, unpaired Student's $t$-test.
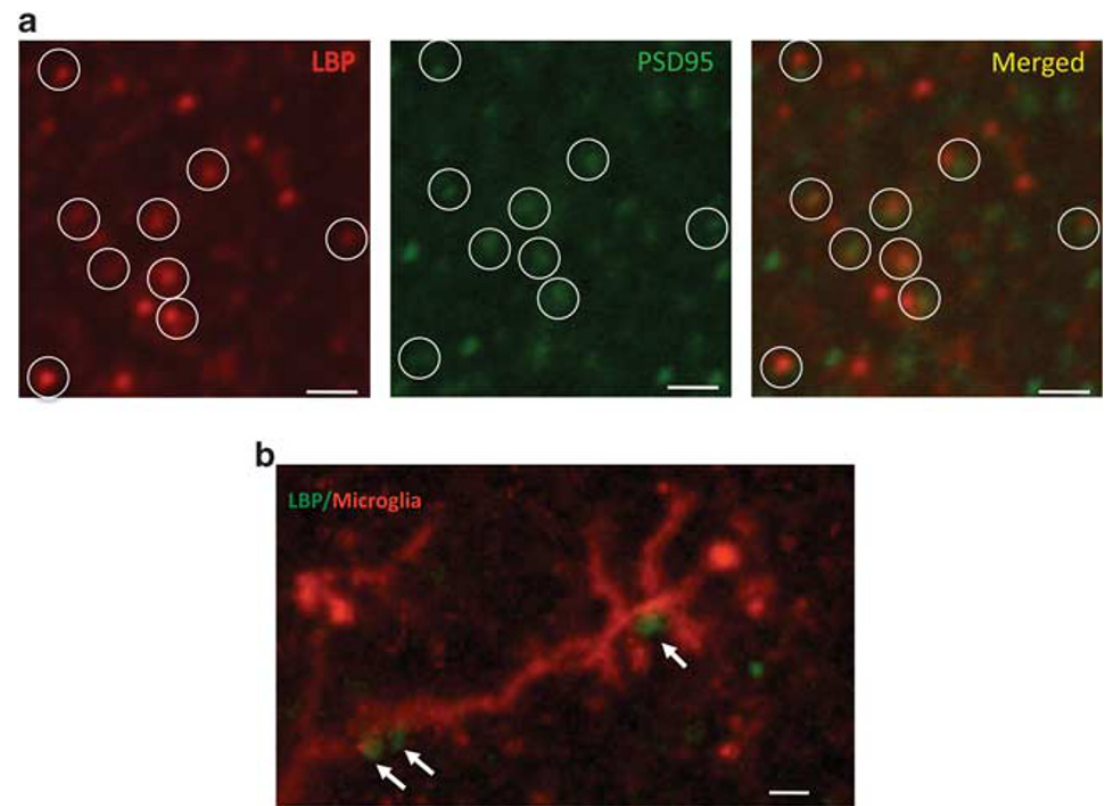

Figure 5 LBP puncta in the developing hippocampus colocalize with the synaptic marker PSD95 and form contact with processes of microglia cells. (a) Confocal image showing that LBP puncta (red) are colocalized with PSD95 puncta (green) in the CAI region of PND 21 male pup. Note that this pattern of LBP staining was seen using several anti-LBP antibodies and was not seen in LBP k.o. mice (Supplementary Figure S3). (b) Confocal image showing LBP puncta (green) making several contacts with microglia processes (red) stained with anti-lba I antibodies. All scale bars are I $\mu$ m.

Previous work has shown that events early in life are able to program expression of genes in the adult brain that are implicated in the regulation of stress reactivity and maternal behavior (Champagne et al, 2006; Murgatroyd et al, 2009; Weaver et al, 2004). These findings suggest that early life stress may influence adult behavior by inducing long-term 
a

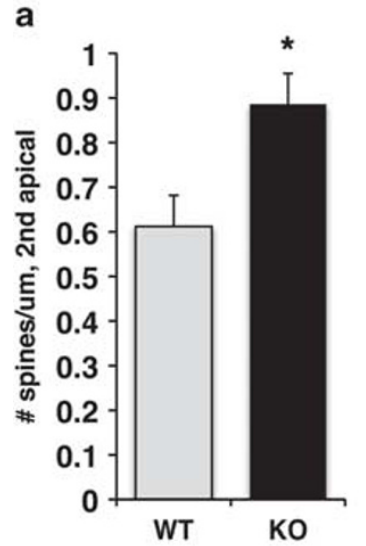

b

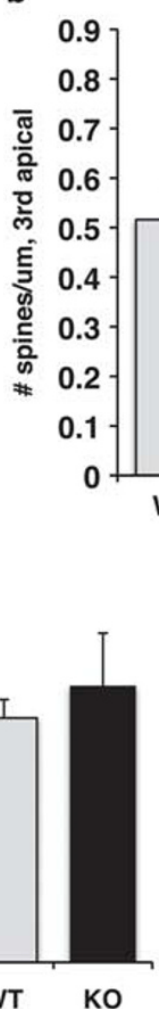

C

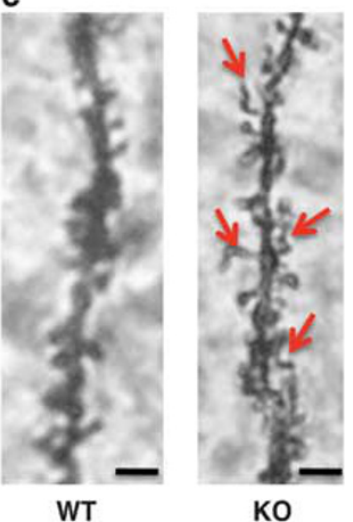

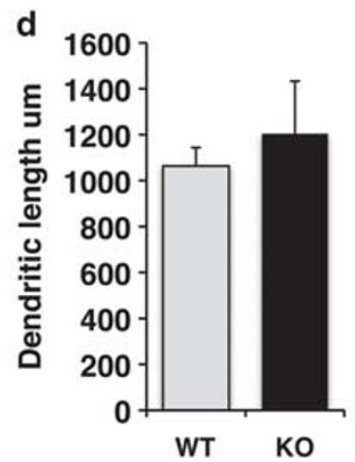

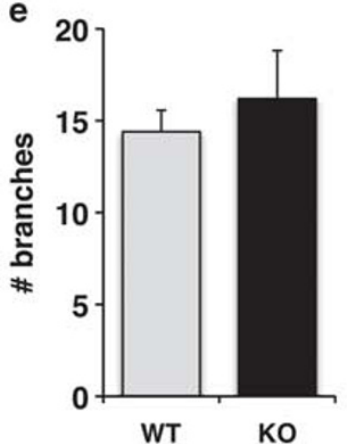

Figure 6 Spine density is increased in juvenile LBP k.o. offspring compared with their wild-type littermates. Spine density in secondary (a) and tertiary (b) apical dendrites of CAI pyramidal neurons was increased in PND 30 LBP k.o. male mice compared with wild-type littermates. (c) Representative Golgi staining of secondary apical dendrite in LBP K.o. and wild-type littermate. Red arrows indicate spines with abnormal morphology. Deletion of LBP had no effect on total dendritic length (d) or number of dendritic branches (e). Results are expressed as mean values \pm SEM, $* p<0.05$, *** $<0.0$ I, paired Student's t-test. Scale bars in (c) are I $\mu \mathrm{m}$.
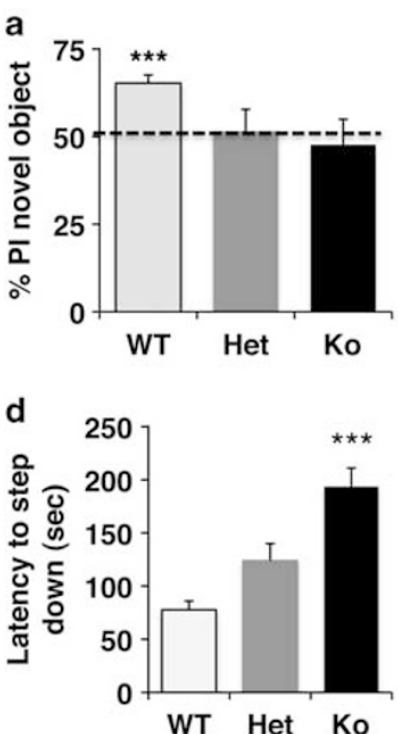
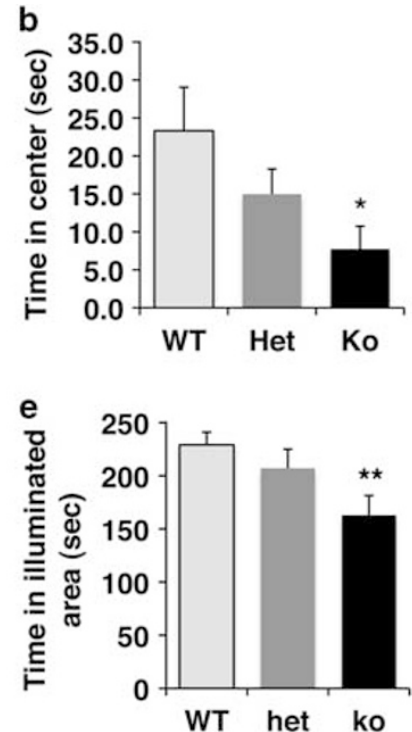
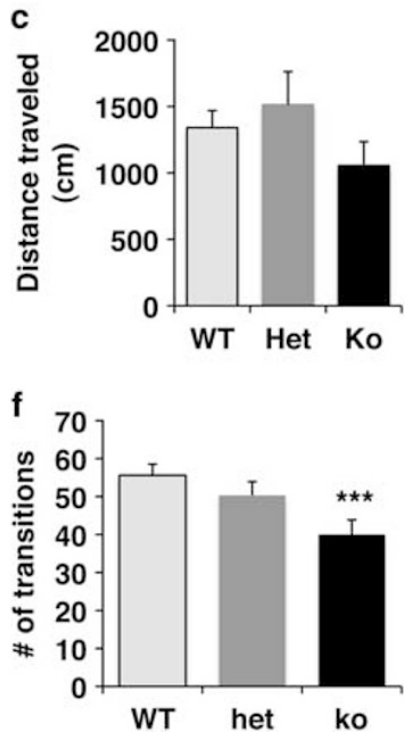

Figure 7 LBP k.o. mice show impairment in hippocampal-dependent learning and increased anxiety-like behavior, which resemble those seen in mice exposed to BDS. (a) Wild-type mice (WT, $n=11$ ), but not heterozygous (Het, $n=10$ ), or knockout (k.o., $n=9$ ) adult males showed the normal tendency to explore a novel object in the object recognition task. Deletion of LBP showed a dose-dependent increase in anxiety-like behavior in the open-field test (b, c), step-down test (d), and dark-light choice (e, f). Graphs represent mean values \pm SEM, one-sample Student's t-test (a) and one-way ANOVA followed by Tukey-HSD post hoc analysis in $(b-f), * p<0.05$, $* * 0<0.01$, **** $p<0.001$ 
changes in gene expression, and led to an intensive search for additional genes whose expression in adulthood is modified by early life stress (Benekareddy et al, 2010; Bordner et al, 2011; Erabi et al, 2007; Kohda et al, 2006; van Heerden et al, 2009; Weaver et al, 2006). Here, we hypothesize that in addition to this well-described longterm alteration in gene expression, some long-term consequences of early life stress are due to transient changes in gene expression that disrupt key neurodevelopmental processes that are in turn necessary to support normal brain function and behavior in adulthood. We used a whole-genome microarray screen to identify genes whose expression is modified by BDS in the hippocampus of 21-day-old pups. This design allowed us to expand the search for neurodevelopmental pathways affected by early life stress that might otherwise be missed by the more traditional search for pathways whose expression is altered in the adult brain. Indeed, the identification of LBP as a gene whose expression is modified by BDS during a critical period of development, but not in adulthood (Figure 3), demonstrates the potential of this strategy to map novel developmental pathways affected by stress, providing new insights into the mechanisms by which early life stress modifies brain function and behavior in adulthood.

\section{LBP Expression Is Inhibited by Early Life Stress}

LBP is a secreted, acute-phase reactant, whose expression in hepatocytes is induced 10-50-fold in response to bacterial infection (Zweigner et al, 2006). LBP k.o. mice have higher mortality when exposed to bacterial infection, demonstrating that LBP serves a critical function in mounting an effective immune response (Jack et al, 1997). LBP function has traditionally been studied in the context of its ability to bind the LPS endotoxin found on the outer membrane of gram-negative bacteria. However, recent work showed that LBP is capable of binding a host of other surface molecules present on gram-positive bacteria, spirochetes, mycobacteria, and mycoplasmas, suggesting a broader function in activating the innate immune system (Zweigner et al, 2006). LBP facilitates removal of foreign pathogens in at least two ways. First, LBP can deliver pathogens for phagocytosis via its ability to bind CD14 receptors found on macrophages (Peterson et al, 1995; Wright et al, 1989). Second, the LBPLPS-CD14 complex can also activate Toll-like receptor 4 found on macrophages and induce the release of proinflammatory cytokines that in turn recruit neutrophils to the site of bacterial infection (Aderem and Ulevitch, 2000; Chow et al, 1999).

Most studies to date have focused on LBP's role in recruiting and activating the innate immune system in response to bacterial infection with little attention paid to its role in non-infectious normal physiological processes. For example, LBP shares high sequence homology with other lipid-transfer proteins and is bound to Apo-A containing chylomicron particles (Wurfel et al, 1994), suggesting that LBP may also serve a function in lipid metabolism. This is interesting because it raises the possibility that synaptic LBP may have a role in lipid insertion necessary for normal spine maturation. In addition, LBP mRNA and protein levels rise in breast tissue that is undergoing involution (Stein et al, 2004). Involution is a process initiated after pup weaning in which elaborated alveolar epithelium in breast tissue undergoes apoptosis in response to pup weaning and the accumulation of excess milk in the mammary glands. Induction of LBP occurs in the absence of an infectious agent and is followed by a significant increase in the number of macrophages and neutrophils present in the breast tissue during later phases of involution. These observations suggest that induction of LBP may facilitate tissue reorganization in a manner analogous to that seen in wound healing (Stein et al, 2004).

Our array findings raised the possibility that LBP might serve an important developmental function that is disturbed by exposure to BDS. We first confirmed the array results using both QPCR and in situ hybridization and replicated them in an independent cohort of 14-day-old pups exposed to BDS. In addition, we showed that exposure to a single, $3 \mathrm{~h}$ maternal separation in 14-day-old pups is sufficient to decrease LBP mRNA and protein levels in the hippocampus. These findings demonstrate that pup-dam separation during the second and third weeks of life causes robust and rapid decline in LBP levels in the hippocampus. Acute maternal separation decreased levels of LBP in the hippocampus but not in the plasma, suggesting that the behavioral effects of BDS are mediated by its ability to regulate expression of LBP in the brain and not in the periphery. In addition, levels of LBP in the plasma are roughly 500 times higher compared with LBP levels in the CSF (Weber et al, 2003), suggesting that plasma LBP cannot readily cross the blood-brain barrier.

The following findings suggest that elevated corticosterone levels, induced by separating the pups from the dam, directly regulate expression of LBP in the hippocampus. First, LBP levels in the hippocampus are highly correlated with plasma corticosterone levels after acute, $3 \mathrm{~h}$ maternal separation. Second, exposure to BDS does not decrease LBP levels in PND 8 pups when the HPA axis has not matured, nor in adult animals when stress is no longer present. Finally, the LBP promoter contains a glucocorticoidreceptor binding site (Schumann et al, 1996), providing a possible mechanism to account for this transcriptional regulation. Nevertheless, additional work is needed to directly test whether acute administration of corticosterone to 14-day-old pups is sufficient to decrease LBP levels in the hippocampus.

\section{LBP Is Required for Normal Hippocampal Development and Function in Adulthood}

Several observations suggest that induction of LBP expression in the developing hippocampus is required for normal hippocampus-dependent function in adulthood. First, expression of LBP in the hippocampus peaks during the second and third weeks of life, a period characterized by increased recruitment of microglia and intense synaptic pruning in the hippocampus (Dalmau et al, 1998; Faulkner et al, 2007). Second, immunohistochemical staining showed that LBP forms a punctate pattern that colocalizes with the synaptic marker PSD95, suggesting that LBP is localized to the developing synapse. Third, LBP puncta are found in close proximity to microglia processes, consistent with its ability to bind CD14 receptors found on microglia cells (Peterson et al, 1995; Wright et al, 1989). Third, LBP k.o. 
mice show abnormal spine morphology, increased spine density, and impaired hippocampal-dependent memory in adulthood. These findings describe a novel role for LBP in neurodevelopment and are consistent with previous work showing that the innate immune system has a critical role in normal neurodevelopment (Stevens et al, 2007).

\section{LBP and the Behavioral Consequences of Early Life Stress}

Here, we propose that some of the behavioral sequelae of BDS are mediated by a decrease in LBP levels in the hippocampus during the second and third weeks of life (for a working model describing this hypothesis, see Figure 8). In support of this hypothesis, we show that LBP expression is necessary for normal spine development in the dorsal hippocampus and that LBP k.o. mice show impaired hippocampal-dependent memory and increased anxietylike behavior in a manner that resembles that seen in animals exposed to BDS. In addition, deletion of similar members of the innate immune system is associated with impairment in synaptic pruning (Stevens et al, 2007). Second, disruption of dam-pup interactions (eg, handling or maternal separation) during the postnatal period is known to increase corticosterone and work from several groups has shown that these manipulations are associated with increased spine density in limbic areas that persist into adulthood (Helmeke et al, 2001; Muhammad and Kolb, 2011; Norrholm and Ouimet, 2001; Ovtscharoff et al, 2006; Poeggel et al, 2003). These data are consistent with the notion that early life stress may interfere with normal synaptic pruning. We propose that this increase in spine density is mediated by a decrease in LBP expression during this critical period of development. One attractive feature of this hypothesis is that it provides a simple mechanism to explain how exposure to stress early in life could modify brain structure and function in adulthood. This is due to the fact that the mammalian brain wires itself by initially forming an overly interconnected rudimentary grid that is then refined in an activity-dependent manner to remove non-functional, redundant, or inappropriate synaptic input (Katz and Shatz, 1996). These initial steps occur at different times in different brain regions and are essential for defining the critical period for each particular brain region. After the critical period has passed, the maturing brain deposits extracellular matrix substances that prevent substantial remodeling of synaptic connections from occurring later in life, though some level of plasticity is maintained throughout life (Hensch and Fagiolini, 2005). Thus, processes that interfere with normal synaptic development early in life persist into adulthood and can modify brain function and behavior later in life, with the monocular deprivation paradigm being one of the best-characterized examples of this phenomenon (Kaffman and Meaney, 2007).

Many conditions associated with mental retardation are characterized by abnormal synaptic morphology and increased spine density (Chechlacz and Gleeson, 2003; Neul and Zoghbi, 2004), underscoring the importance of appropriate synaptic pruning for normal brain functioning in adulthood. This issue has been studied extensively in Fragile $\mathrm{X}$ syndrome where postmortem analysis has shown increased density of immature spines in individuals with this syndrome (Hinton et al, 1991; Irwin et al, 2001; Rudelli

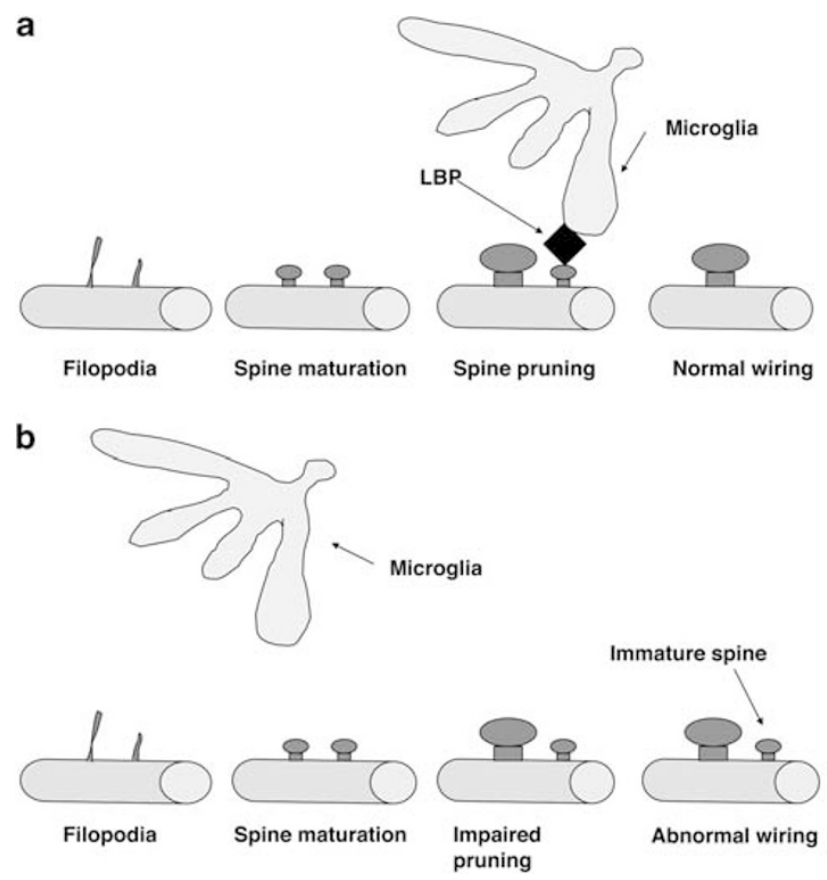

Figure 8 Schematic model for LBP function. (a, top) Under normal developmental conditions, LBP (shown in black) marks non-functional synapses and targets them for elimination by its ability to recruit microglia. (b, bottom) Synaptic pruning is impaired under conditions in which LBP levels are abnormally low such as observed in animals exposed to early life stress or genetic deletion of LBP. Abnormal pruning during a critical period leads to abnormal synaptic wiring that persists into adulthood and affects complex behaviors.

et al, 1985), a finding that was replicated in studies examining this issue in the mouse k.o. model (Comery et al, 1997; Dolen et al, 2007; Restivo et al, 2005). Increased spine density has also been reported in other conditions associated with mental retardation such as Down's syndrome and phenylketonuria (Chechlacz and Gleeson, 2003).

\section{Study Limitations}

This study has several limitations. First, the constitutive deletion of LBP across all tissues and ages does not allow us to conclusively conclude that the behavioral abnormalities seen in LBP k.o. are due to its absence in the hippocampus during the second and third weeks of life. This is especially true given that LBP is highly expressed in the choroid plexus (Supplementary Figure 2) raising the possibility that some of the behavioral sequelae of BDS or its deletion in LBP k.o. might be mediated by its action in the choroid plexus. Nevertheless, the findings that LBP is localized to the developing synapse in the hippocampus and that its deletion causes an increase in spine density in CA1 neurons and poor hippocampal function provide compelling evidence for a direct function of LBP in the developing hippocampus. Second, we have not tested yet whether exposure to BDS is associated with increased spine density. However, a large body of work from several groups has consistently shown that disruption of pup-dam interactions early in life is associated with increased spine density that persists into adulthood (Helmeke et al, 2001; Muhammad and Kolb, 2011; Norrholm and Ouimet, 2001; Ovtscharoff 
et al, 2006; Poeggel et al, 2003). Third, given the somewhat unusual stress reactivity that characterizes the BALB/cByj strain (Shanks et al, 1990; Zaharia et al, 1996) additional work is needed to confirm our findings in other strains of mice. Nonetheless, our findings that BDS decreases expression of Transthyretin (TTR) and Autotaxin (ENPP2, see Supplementary Table S1) are consistent with previous work examining this issue in the adult rat brain (Kohda et al, 2006) and suggest that some of our findings are robust across different species. Fourth, additional work is needed to validate more genes found on the array and examine their role in mediating some of the developmental effects of BDS. For example, three of the five most highly regulated genes found on the array are genes that regulate neural stem cell proliferation and survival (eg, ECRG, Kujuro et al, 2010; ENPP2, Koike et al, 2011; SOSTDC1, Lintern et al, 2009), providing a possible molecular explanation for the reduced DNA content seen in the hippocampus of pups exposed to BDS (Wei et al, 2010). Finally, our data does not allow us to assess the exact contribution that a decrease in LBP expression in the hippocampus has in the behavioral sequelae of animals exposed to BDS, nor do we imply that all the behavioral consequences of BDS are mediated by its ability to inhibit expression of LBP. Instead, we suggest that exposure to BDS modifies many developmental pathways with LBP being one of the most highly regulated pathway affected by this manipulation.

\section{CONCLUSIONS}

Our findings describe a novel role for LBP in hippocampal development and function in adulthood, and are consistent with a large body of work showing that the innate immune system has a critical role in synaptic pruning during a critical period of development. The findings that LBP expression is dramatically decreased in response to stress early in life and that LBP k.o. animals show behavioral abnormalities that resemble those seen in animals exposed to early life stress raise the possibility that some of the behavioral consequences of early life stress are mediated by its ability to impair normal activation of the innate immune system during a critical period of development.

\section{ACKNOWLEDGEMENTS}

We thank E Cumberbatch, R Duman, and M Picciotto for helpful comments on the manuscript. This work was supported by NIMH 1KO8MH074856, NARSAD young investigator award 2007, DANA foundation Program in Brain and Immuno-imagine 2011, and the Clinical Neuroscience Division of the VA National Center for PTSD. AS was supported by NIA grant P01 AG 030004-01A1, NIA/ AFAR grant AG030970, and a grant from the Yale Pepper Center. We also wish to acknowledge the support of the Yale Center for High Performance Computation in Biology and Biomedicine and NIH grant RR19895, which funded the computing instrumentation.

\section{DISCLOSURE}

The authors declare no conflict of interest.

\section{REFERENCES}

Aderem A, Ulevitch RJ (2000). Toll-like receptors in the induction of the innate immune response. Nature 406: 782-787.

Aisa B, Tordera R, Lasheras B, Del Rio J, Ramirez MJ (2007). Cognitive impairment associated to HPA axis hyperactivity after maternal separation in rats. Psychoneuroendocrinology 32: 256-266.

Benekareddy M, Goodfellow NM, Lambe EK, Vaidya VA (2010). Enhanced function of prefrontal serotonin 5-HT(2) receptors in a rat model of psychiatric vulnerability. J Neurosci 30: 12138-12150.

Berrebi AS, Fitch RH, Ralphe DL, Denenberg JO, Friedrich Jr VL, Denenberg VH (1988). Corpus callosum: region-specific effects of sex, early experience and age. Brain Res 438: 216-224.

Bordner KA, George ED, Carlyle BC, Duque A, Kitchen RR, Lam TT et al (2011). Functional genomic and proteomic analysis reveals disruption of myelin-related genes and translation in a mouse model of early life neglect. Front Psychiatry 2: 18.

Boulanger LM, Shatz CJ (2004). Immune signalling in neural development, synaptic plasticity and disease. Nat Rev Neurosci 5: 521-531.

Champagne DL, Bagot RC, van Hasselt F, Ramakers G, Meaney MJ, de Kloet ER et al (2008). Maternal care and hippocampal plasticity: evidence for experience-dependent structural plasticity, altered synaptic functioning, and differential responsiveness to glucocorticoids and stress. J Neurosci 28: 6037-6045.

Champagne FA, Weaver IC, Diorio J, Dymov S, Szyf M, Meaney MJ (2006). Maternal care associated with methylation of the estrogen receptor- $\mid\{$ alphal\}1b promoter and estrogen receptor$\backslash$ alphal\} expression in the medial preoptic area of female offspring. Endocrinology 147: 2909-2915.

Chechlacz M, Gleeson JG (2003). Is mental retardation a defect of synapse structure and function? Pediatr Neurol 29: 11-17.

Chow JC, Young DW, Golenbock DT, Christ WJ, Gusovsky F (1999). Toll-like receptor-4 mediates lipopolysaccharide-induced signal transduction. J Biol Chem 274: 10689-10692.

Comery TA, Harris JB, Willems PJ, Oostra BA, Irwin SA, Weiler IJ et al (1997). Abnormal dendritic spines in fragile X knockout mice: maturation and pruning deficits. Proc Natl Acad Sci USA 94: 5401-5404.

Dalmau I, Finsen B, Zimmer J, Gonzalez B, Castellano B (1998). Development of microglia in the postnatal rat hippocampus. Hippocampus 8: 458-474.

Denenberg VH, Rosen GD, Hofmann M, Gall J, Stockler J, Yutzey DA (1981). Neonatal postural asymmetry and sex differences in the rat. Brain Res 254: 417-419.

Dolen G, Osterweil E, Rao BS, Smith GB, Auerbach BD, Chattarji S et al (2007). Correction of fragile X syndrome in mice. Neuron 56: 955-962.

Duric V, Banasr M, Licznerski P, Schmidt HD, Stockmeier CA, Simen AA et al (2010). A negative regulator of MAP kinase causes depressive behavior. Nat Med 16: 1328-1332.

Erabi K, Morinobu S, Kawano K, Tsuji S, Yamawaki S (2007). Neonatal isolation changes the expression of IGF-IR and IGFBP2 in the hippocampus in response to adulthood restraint stress. Int J Neuropsychopharmacol 10: 369-381.

Fabricius K, Wortwein G, Pakkenberg B (2008). The impact of maternal separation on adult mouse behaviour and on the total neuron number in the mouse hippocampus. Brain Struct Funct 212: 403-416.

Faulkner RL, Low LK, Cheng HJ (2007). Axon pruning in the developing vertebrate hippocampus. Dev Neurosci 29: 6-13.

George ED, Bordner KA, Elwafi HM, Simen AA (2011). Maternal separation with early weaning: a novel mouse model of early life neglect. BMC Neurosci 11: 123.

Gilbert R, Widom CS, Browne K, Fergusson D, Webb E, Janson S (2009). Burden and consequences of child maltreatment in high-income countries. Lancet 373: 68-81. 
Green JG, McLaughlin KA, Berglund PA, Gruber MJ, Sampson NA, Zaslavsky AM et al (2010). Childhood adversities and adult psychiatric disorders in the national comorbidity survey replication I: associations with first onset of DSM-IV disorders. Arch Gen Psychiatry 67: 113-123.

Helmeke C, Poeggel G, Braun K (2001). Differential emotional experience induces elevated spine densities on basal dendrites of pyramidal neurons in the anterior cingulate cortex of Octodon degus. Neuroscience 104: 927-931.

Hensch TK, Fagiolini M (2005). Excitatory-inhibitory balance and critical period plasticity in developing visual cortex. Prog Brain Res 147: 115-124.

Hinton VJ, Brown WT, Wisniewski K, Rudelli RD (1991). Analysis of neocortex in three males with the fragile $\mathrm{X}$ syndrome. Am J Med Genet 41: 289-294.

Irwin SA, Patel B, Idupulapati M, Harris JB, Crisostomo RA, Larsen $\mathrm{BP}$ et al (2001). Abnormal dendritic spine characteristics in the temporal and visual cortices of patients with fragile-X syndrome: a quantitative examination. Am J Med Genet 98: 161-167.

Jack RS, Fan X, Bernheiden M, Rune G, Ehlers M, Weber A et al (1997). Lipopolysaccharide-binding protein is required to combat a murine gram-negative bacterial infection. Nature 389: $742-745$.

Kaffman A (2009). The silent epidemic of neurodevelopmental injuries. Biol Psychiatry 66: 624-626.

Kaffman A, Meaney M (2007). Neurodevelopmental sequelae of postnatal maternal care in rodents: clinical and research implications of molecular insights. J Child Psychol Psychiatry 48: 224-244.

Kalinichev M, Easterling KW, Holtzman SG (2002). Early neonatal experience of Long-Evans rats results in long-lasting changes in reactivity to a novel environment and morphine-induced sensitization and tolerance. Neuropsychopharmacology 27: 518-533.

Katz LC, Shatz CJ (1996). Synaptic activity and the construction of cortical circuits. Science 274: 1133-1138.

Kikusui T, Nakamura K, Kakuma Y, Mori Y (2006). Early weaning augments neuroendocrine stress responses in mice. Behav Brain Res 175: 96-103.

Kohda K, Jinde S, Iwamoto K, Bundo M, Kato N, Kato T (2006). Maternal separation stress drastically decreases expression of transthyretin in the brains of adult rat offspring. Int $J$ Neuropsychopharmacol 9: 201-208.

Koike S, Yutoh Y, Keino-Masu K, Noji S, Masu M, Ohuchi H (2011). Autotaxin is required for the cranial neural tube closure and establishment of the midbrain-hindbrain boundary during mouse development. Dev Dyn 240: 413-421.

Kujuro Y, Suzuki N, Kondo T (2010). Esophageal cancer-related gene 4 is a secreted inducer of cell senescence expressed by aged CNS precursor cells. Proc Natl Acad Sci USA 107: 8259-8264.

Levine S (1994). The ontogeny of the hypothalamic-pituitaryadrenal axis. The influence of maternal factors. Ann NY Acad Sci 746: 275-288; discussion 289-293.

Lintern KB, Guidato S, Rowe A, Saldanha JW, Itasaki N (2009). Characterization of wise protein and its molecular mechanism to interact with both Wnt and BMP signals. J Biol Chem 284: 23159-23168.

Millstein RA, Holmes A (2007). Effects of repeated maternal separation on anxiety- and depression-related phenotypes in different mouse strains. Neurosci Biobehav Rev 31: 3-17.

Millstein RA, Ralph RJ, Yang RJ, Holmes A (2006). Effects of repeated maternal separation on prepulse inhibition of startle across inbred mouse strains. Genes Brain Behav 5: 346-354.

Muhammad A, Kolb B (2011). Maternal separation altered behavior and neuronal spine density without influencing amphetamine sensitization. Behav Brain Res 223: 7-16.

Murgatroyd C, Patchev AV, Wu Y, Micale V, Bockmuhl Y, Fischer $D$ et al (2009). Dynamic DNA methylation programs persistent adverse effects of early-life stress. Nat Neurosci 12: 1559-1566.
Nemeroff CB, Heim CM, Thase ME, Klein DN, Rush AJ, Schatzberg $\mathrm{AF}$ et al (2003). Differential responses to psychotherapy vs pharmacotherapy in patients with chronic forms of major depression and childhood trauma. Proc Natl Acad Sci USA 100: 14293-14296.

Neul JL, Zoghbi HY (2004). Rett syndrome: a prototypical neurodevelopmental disorder. Neuroscientist 10: 118-128.

Norrholm SD, Ouimet CC (2001). Altered dendritic spine density in animal models of depression and in response to antidepressant treatment. Synapse 42: 151-163.

Ovtscharoff Jr W, Helmeke C, Braun K (2006). Lack of paternal care affects synaptic development in the anterior cingulate cortex. Brain Res 1116: 58-63.

Parfitt DB, Levin JK, Saltstein KP, Klayman AS, Greer LM, Helmreich DL (2004). Differential early rearing environments can accentuate or attenuate the responses to stress in male C57BL/6 mice. Brain Res 1016: 111-118.

Peterson PK, Gekker G, Hu S, Sheng WS, Anderson WR, Ulevitch $\mathrm{RJ}$ et al (1995). CD14 receptor-mediated uptake of nonopsonized Mycobacterium tuberculosis by human microglia. Infect Immun 63: $1598-1602$.

Pfaffl MW (2001). A new mathematical model for relative quantification in real-time RT-PCR. Nucleic Acids Res 29: e45.

Poeggel G, Helmeke C, Abraham A, Schwabe T, Friedrich P, Braun K (2003). Juvenile emotional experience alters synaptic composition in the rodent cortex, hippocampus, and lateral amygdala. Proc Natl Acad Sci USA 100: 16137-16142.

Priebe K, Romeo RD, Francis DD, Sisti HM, Mueller A, McEwen BS et al (2005). Maternal influences on adult stress and anxiety-like behavior in C57BL/6J and BALB/cJ mice: a cross-fostering study. Dev Psychobiol 47: 398-407.

Ren-Patterson RF, Cochran LW, Holmes A, Lesch KP, Lu B, Murphy DL (2006). Gender-dependent modulation of brain monoamines and anxiety-like behaviors in mice with genetic serotonin transporter and BDNF deficiencies. Cell Mol Neurobiol 26: $755-780$.

Restivo L, Ferrari F, Passino E, Sgobio C, Bock J, Oostra BA et al (2005). Enriched environment promotes behavioral and morphological recovery in a mouse model for the fragile $\mathrm{X}$ syndrome. Proc Natl Acad Sci USA 102: 11557-11562.

Rice CJ, Sandman CA, Lenjavi MR, Baram TZ (2008). A novel mouse model for acute and long-lasting consequences of early life stress. Endocrinology 149: 4892-4900.

Romeo RD, Mueller A, Sisti HM, Ogawa S, McEwen BS, Brake WG (2003). Anxiety and fear behaviors in adult male and female C57BL/6 mice are modulated by maternal separation. Horm Behav 43: 561-567.

Rudelli RD, Brown WT, Wisniewski K, Jenkins EC, LaureKamionowska M, Connell F et al (1985). Adult fragile X syndrome. Clinico-neuropathologic findings. Acta Neuropathol 67: 289-295.

Schumann RR, Kirschning CJ, Unbehaun A, Aberle HP, Knope HP, Lamping $\mathrm{N}$ et al (1996). The lipopolysaccharide-binding protein is a secretory class 1 acute-phase protein whose gene is transcriptionally activated by APRF/STAT/3 and other cytokine-inducible nuclear proteins. Mol Cell Biol 16: 3490-3503.

Shanks N, Griffiths J, Zalcman S, Zacharko RM, Anisman H (1990). Mouse strain differences in plasma corticosterone following uncontrollable footshock. Pharmacol Biochem Behav 36: 515-519.

Stein T, Morris JS, Davies CR, Weber-Hall SJ, Duffy MA, Heath VJ et al (2004). Involution of the mouse mammary gland is associated with an immune cascade and an acute-phase response, involving LBP, CD14 and STAT3. Breast Cancer Res 6: R75-R91.

Stevens B, Allen NJ, Vazquez LE, Howell GR, Christopherson KS, Nouri $\mathrm{N}$ et al (2007). The classical complement cascade mediates CNS synapse elimination. Cell 131: 1164-1178. 
van Heerden JH, Conesa A, Stein DJ, Montaner D, Russell V, Illing $\mathrm{N}$ (2009). Parallel changes in gene expression in peripheral blood mononuclear cells and the brain after maternal separation in the mouse. BMC Res Notes 2: 195.

Weaver IC, Cervoni N, Champagne FA, D'Alessio AC, Sharma S, Seckl JR et al (2004). Epigenetic programming by maternal behavior. Nat Neurosci 7: 847-854.

Weaver IC, Meaney MJ, Szyf M (2006). Maternal care effects on the hippocampal transcriptome and anxiety-mediated behaviors in the offspring that are reversible in adulthood. Proc Natl Acad Sci USA 103: 3480-3485.

Weber JR, Freyer D, Alexander C, Schroder NW, Reiss A, Kuster C et al (2003). Recognition of pneumococcal peptidoglycan: an expanded, pivotal role for LPS binding protein. Immunity 19: 269-279.

Wei L, David A, Duman RS, Anisman H, Kaffman A (2010). Early life stress increases anxiety-like behavior in Balbc mice despite a compensatory increase in levels of postnatal maternal care. Horm Behav 57: 396-404.
Wright SD, Tobias PS, Ulevitch RJ, Ramos RA (1989). Lipopolysaccharide (LPS) binding protein opsonizes LPS-bearing particles for recognition by a novel receptor on macrophages. J Exp Med 170: 1231-1241.

Wurfel MM, Kunitake ST, Lichenstein H, Kane JP, Wright SD (1994). Lipopolysaccharide (LPS)-binding protein is carried on lipoproteins and acts as a cofactor in the neutralization of LPS. J Exp Med 180: 1025-1035.

Zaharia MD, Kulczycki J, Shanks N, Meaney MJ, Anisman H (1996). The effects of early postnatal stimulation on Morris water-maze acquisition in adult mice: genetic and maternal factors. Psychopharmacology (Berl) 128: 227-239.

Zeanah CH, Egger HL, Smyke AT, Nelson CA, Fox NA, Marshall PJ et al (2009). Institutional rearing and psychiatric disorders in romanian preschool children. Am J Psychiatry 166: 777-785.

Zweigner J, Schumann RR, Weber JR (2006). The role of lipopolysaccharide-binding protein in modulating the innate immune response. Microbes Infect 8: 946-952.

Supplementary Information accompanies the paper on the Neuropsychopharmacology website (http://www.nature.com/npp) 